\title{
Correction to: Factors Contributing to Psychological III\#Effects and Resilience of Caregivers of Children with Developmental Disabilities During a Nation\#wide Lockdown During the COVID\#19 Pandemic
}

\author{
Tammy S. H. Lim ${ }^{1,2} \cdot$ Mae Yue Tan ${ }^{1,2} \cdot$ Ramkumar Aishworiya $^{1,2} \cdot$ Ying Qi Kang ${ }^{1,2} \cdot$ Magdalena Yvonne Koh $^{1,2}$. \\ Liang Shen ${ }^{3} \cdot$ Shang Chee Chong ${ }^{1,2}$
}

Published online: 26 July 2021

(C) Springer Science+Business Media, LLC, part of Springer Nature 2021

\section{Correction to: \\ Journal of Autism and Developmental Disorders \\ https://doi.org/10.1007/s10803-021-05180-9}

In this article the author name Ramkumar Aishworiya was incorrectly written as Aishworiya Ramkumar.

The Original article has been updated.

Publisher's Note Springer Nature remains neutral with regard to jurisdictional claims in published maps and institutional affiliations.

The original article can be found online at https://doi.org/10.1007/ s10803-021-05180-9.

Mae Yue Tan

mae_yue_tan@nuhs.edu.sg

1 Child Development Unit, Khoo Teck Puat-National University Children's Medical Institute, National University Health System, Singapore, Singapore

2 Department of Paediatrics, Yong Loo Lin School of Medicine, National University of Singapore, Singapore, Singapore

3 Biostatistics Unit, Yong Loo Lin School of Medicine, National University of Singapore, Singapore, Singapore 